\title{
Erratum to: Boronate-affinity based magnetic molecularly imprinted nanoparticles for the efficient extraction of the model glycoprotein horseradish peroxidase
}

\author{
Xiao-Yu Sun $^{1,2} \cdot$ Run-Tian Ma ${ }^{1} \cdot$ Juan Chen $^{1} \cdot$ Yan-Ping Shi ${ }^{1}$
}

Published online: 1 August 2017

(C) Springer-Verlag GmbH Austria 2017

\section{Erratum to: Microchimica Acta}

\section{DOI: 10.1007/s00604-017-2373-2}

The original version of this article unfortunately contained a mistake in the Acknowledgements section. Given in this article is the correct acknowledgement statement.

Acknowledgements Financial supports from the National Natural Science Foundation of China (No. 21575150, 21375136), CAS President's International Fellowship Initiative (SL: 191), the funds for Distinguished Young Scientists of Gansu (1506RJDA281) and the top priority program of "One-Three-Five" Strategic Planning of Chinese Academy of Sciences are gratefully acknowledged.

Juan Chen

chenjuan@licp.cas.cn

$\bowtie$ Yan-Ping Shi

shiyp@licp.cas.cn

1 Key Laboratory of Chemistry of Northwestern Plant Resources and Key Laboratory for Natural Medicine of Gansu Province, Lanzhou Institute of Chemical Physics, Chinese Academy of Sciences,

Lanzhou 730000, China

2 University of Chinese Academy of Sciences, Chinese Academy of Sciences, Beijing 100049, People's Republic of China 